\title{
Optimizing Mechanical Ventilation in the Neonatal ICU
}

The history of pulmonary management of the neonatal patient has been marked by substantial reductions in morbidity and mortality. The general application of mechanical ventilation, the instillation of surfactant into the trachea, and CPAP have each contributed in important ways. Beyond the introduction of these modalities to neonatal care is an area where additional reductions in morbidity occur.

This is the realm in which the meticulous application of the scientific method incrementally optimizes the general applications for improved outcomes. As important as the work of the practicing clinician in the saving of lives is the work of the medical researcher. Carefully conducted and published research can result in a distributed change in practice that will reduce morbidity and mortality across the world.

In this issue of Respiratory CARe, the study by Ünal et $\mathrm{l}^{1}$ regarding volume-guarantee ventilation demonstrates the steady scientific work to find improvements in neonatal mechanical ventilation. In the early decades of neonatal mechanical ventilation, pneumotachometers with small dead space and adequate sensitivity were not available. ${ }^{2}$ For this reason, neonatal mechanical ventilation was predominately provided using the time-cycled and pressurelimited mode. Exhaled tidal volumes were not monitored. By the mid-1990s, several manufacturers ${ }^{2}$ had developed ventilators capable of providing and measuring tidal volumes as low as $4 \mathrm{~mL}$. The rapid appearance of these new ventilators came with an assortment of hybrid modes of mechanical ventilation. The body of published research regarding neonatal mechanical ventilation prior to the mid1990s was dominated by the study of time-cycled and pressure-limited methodologies. ${ }^{3}$ The introduction of a variety of modes of mechanical ventilation created a space for researchers to apply the power of science to further improve outcomes. A systematic review and meta-analysis demonstrated a reduction in several ventilator-associated morbidities for premature infants when using volume-targeted ventilation compared to traditional pressure-limited

The authors have disclosed no conflicts of interest.

Correspondence: James L Hulse PhD MPH RRT-NPS RPFT, 416 Wimer Street, Ashland, OR 97520. E-mail: james.hulse@ oit.edu.

DOI: $10.4187 /$ respcare.05899 ventilation. ${ }^{4}$ However, more data are needed to evaluate advantages between volume-targeted modes in clinical outcomes. The authors of this study are part of the scientific community who are tackling this challenge. Ventilator synchrony in neonates has been shown to decrease work of

See the Original Study on Page 1525

breathing, improve oxygenation, and demonstrate more consistent tidal volumes..$^{5-7}$ Asynchrony is most often associated with increased use of sedation and longer duration of mechanical ventilation. Methods of improving synchrony optimize breath triggering, flow delivery, and breath cycling.

The authors present a randomized, controlled trial over the course of 2.5 years at a Level III NICU. The subjects consisted of 42 premature infants (24-32 weeks of gestational age) who required mechanical ventilation due to respiratory distress syndrome. The premies were randomly separated into those treated with synchronized intermittent mechanical ventilation with volume guarantee (SIMV + VG) or pressure support ventilation with volume guarantee (PSV+VG). The ventilatory parameters, blood gases, tracheal aspirate cytokines, mortality rate, and risk of chronic lung disease were measured and compared. PSV $+\mathrm{VG}$ was found to produce more breaths with an appropriate tidal volume and fewer breaths with a low tidal volume as compared to SIMV + VG. Along with the increase in more appropriate tidal volumes, there was a significantly lower heart rate in babies ventilated using PSV $+\mathrm{VG}$ compared to the SIMV + VG group. PSV + VG allowed the subjects significant control over triggering, flow delivery, and breath cycling. A significantly lower heart rate of 145 beats/min (range $138-153$ beats/min) compared to 154 beats/min (144162 beats/min) is an important finding.

A lower heart rate suggests a lower metabolic rate, which is associated with a lower level of cardiopulmonary stress. It suggests a better synchronization of this mode of mechanical ventilation compared to the other. This finding is the sort of incremental knowledge that we should take note of.

Analyzing interleukin (IL) from tracheal aspirates is another interesting finding of this article. Lung injury, bronchial microbial colonization, and hyperoxic exposure in- 


\section{EDITORIALS}

duced by mechanical ventilation may contribute to lung inflammation in premies, which can lead to chronic lung disease. ${ }^{8,9}$ In this study, IL- $1 \beta$, which induced the most potent neutrophil chemotactic factor IL-8 in airway epithelial cell, was elevated significantly in both groups. However, the lower rate of chronic lung disease in the PS + VG group was not significant.

Despite the small sample size and the insufficient paired tracheal aspirated samples, studies like this appropriately combined with the findings of other studies can collectively lead to real improvements for the care of our vulnerable patients.

\section{James L Hulse PhD MPH RRT-NPS RPFT Nam H Mai MD PhD RRT}

Oregon Institute of Technology Department of Respiratory Therapy Klamath Falls, Oregon

\section{REFERENCES}

1. Ünal S, Ergenekon E, Aktas S, Beken S, Kazanci E. Effects of volume guarantee ventilation combined with two different modes in preterm infants: randomized controlled trial. Respir Care 2017;62(12):15251532 .
2. Donn S, Boon W. Mechanical ventilation of the neonate: should we target volume or pressure? Respir Care 2009;54(9):1236-43.

3. Bower L, Barnhart S, Betit P, Hendon B, Masi-Lynch J, Wilson B. AARC Clinical Practice Guideline: neonatal time-triggered, pressurelimited, time-cycled mechanical ventilation. Respir Care 1994;39(8): 808-16.

4. Peng W, Zhu H, Shi H, Liu E. Volume-targeted ventilation is more suitable than pressure-limited ventilation for preterm infants: a systematic review and meta-analysis. Arch Dis Child Fetal Neonatal Ed 2014;99(2):F158-F165.

5. Jarreau PH, Moreitte G, Mussat P, Mariette C, Mahanna A, Harf A, Lorino H. Patient-triggered ventilation decreases the work of breathing in neonates. Am J Respir Crit Care Med 1996;153(3):1176-81.

6. Cleary JP, Bernstein G, Mannino FL, Heldt GP. Improved oxygenation during synchronized intermittent mandatory ventilation in neonates with respiratory distress syndrome: a randomized, crossover study. J Pediatr 1995;126(3):407-11.

7. Bernstein G, Heldt GP, Mannino FL. Increased and more consistent tidal volumes during synchronized intermittent mandatory ventilation in newborn infants. Am J Respir Crit Care Med 1994;150(5 Pt 1): 1444-48.

8. Kotecha S, Wilson L, Wangoo A, Silverman M, Shaw RJ. Increase in interleukin (IL)-1 beta and IL-6 in bronchoalveolar lavage fluid obtained from infants with chronic lung disease of prematurity. Pediatr Res 1996;40(2):250-56.

9. Little S, Dean T, Bevin S, et al. Role of elevated plasma soluble ICAM-1 and bronchial lavage fluid IL-8 levels as markers of chronic lung disease in premature infants. Thorax 1995;50(10):1073-79. 